\title{
Diseño e implementación del sistema de monitoreo, supervisión y control automático del proceso de destilación de agua en el área de inyectables de LIFE C.A.
}

\author{
Alan D. Cuenca S. y Milton S. León E.
}

\begin{abstract}
Resumen - El presente artículo describe el diseño e implementación de un sistema desarrollado para el monitoreo, supervisión y control del proceso de destilación de agua, empleando un autómata programable y un panel táctil de marca SIEMENS, además del protocolo de comunicación Industrial Ethernet TCP/IP. Este sistema almacena información recopilada del proceso en una base de datos creada en SQL Server 2005. La interfaz gráfica de la pantalla táctil permite la visualización de los datos y variables más relevantes y generar reportes en hojas de cálculo de Microsoft Excel. Este sistema ha sido orientado para ser utilizado como herramienta que facilite a administradores a conocer la producción y funcionamiento del área de Inyectables y mediante el análisis de los reportes la toma de decisiones.
\end{abstract}

\section{INTRODUCCIÓN}

La imposibilidad de las empresas para tomar decisiones correctas puede ser resultado de la falta de datos confiables relacionados a la productividad industrial. En la actualidad grandes empresas han optado por la utilización de sistemas automatizados así como la implementación de herramientas que permitan un correcto análisis de sus procesos para reducir las pérdidas y por consecuente incrementar los niveles de producción.

La automatización de los procesos de producción consiste en la búsqueda del hardware adecuado para la aplicación, el diseño del software para el cumplimiento de diversas sentencias de control, seleccionar variables de interés real para su almacenamiento como datos históricos y la aplicación de sofisticadas herramientas de análisis,

Alan D. Cuenca S., Milton S. León E., Ingeniería en Electrónica, Automatización y Control, Universidad de las Fuerzas Armadas ESPE, Sangolquí, Ecuador, E-mails: alandcuencas@hotmail.com, milen37@hotmail.com. todo esto se complementa con el desarrollo de bases de datos.

Los procesos automatizados aportan numerosas ventajas a la producción industrial. Un proceso de fabricación automatizada en la industria hoy en día, significa un producto final de mayor calidad y más competitivo debido a factores tales como la normalización de procesos y productos, la velocidad de producción, programación de la producción, la reducción continua de los residuos y menos probabilidades de equivocarse. Evita el contacto del producto con los recursos humanos, cuyos resultados van desde más higiene en el proceso, alta confiabilidad hasta un elevado nivel de calidad del producto final.

El gran desarrollo de la automatización de procesos en la actualidad permite disponer de controladores lógicos programables (PLC's), sistemas SCADA, sistemas de control distribuido, y servidores OPC que junto a paquetes de software capaces de realizar tareas de adquisición, almacenamiento y presentación de datos históricos, ofrecen características de mejoramiento productivo en un alto y sofisticado nivel.

Las principales desventajas de estos sistemas son el elevado costo de implementación debido a la adquisición de hardware y software con licencias de última tecnología que permitan un correcto y eficaz funcionamiento de los mismos.

El desarrollo del sistema de monitoreo, supervisión y control busca obtener un alto nivel de producción industrial en la sección de Inyectables de mayor volumen, así como un sistema confiable y adaptable al medio industrial farmacéutico ecuatoriano.

II. PROCESO DE DESTILACIÓN DE AGUA [1] 
El agua usada en la industria farmacéutica para los inyectables de denomina AGUA INYECTABLE (Water Injection) (Fig. 1)

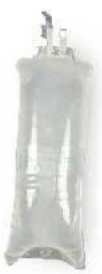

Fig. 1 Agua Inyectable

La destilación de los sistemas de agua farmacéutica es uno de los puntos críticos en la industria, por no decirlo de vital importancia, no solo por las grandes cantidades de agua que se consumen sino también por la naturaleza propia del agua, ya que puede caracterizarse e identificarse por el tipo y cantidad de sustancias que contiene. Estos atributos de calidad no siempre pueden medirse antes de su uso ya que la contaminación microbiana necesita varios días para poder determinarse y durante ese tiempo, el agua se utiliza, los productos se fabrican y los costes suben. La destilación es esencial para prevenir la necesidad de retirar o destruir productos por problemas de contaminación causados por el agua.

En la empresa LIFE el equipo más importante de este proceso es el destilador de agua OLSA QV 2000 (Fig. 2), el cual trabaja automática y continuamente y permite lograr una producción de 2000 lt/h de agua destilada.

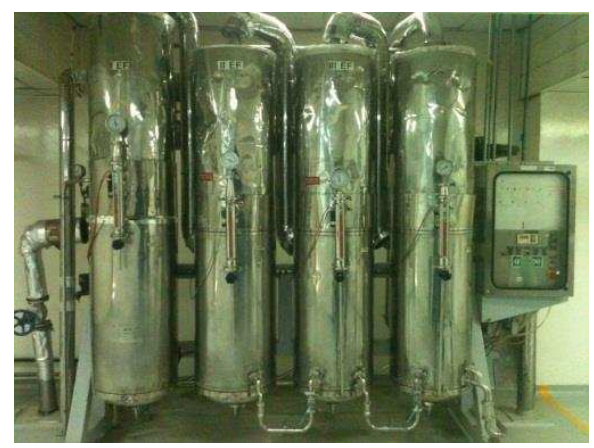

Fig. 2 Destilador de agua OLSA QV 2000

\section{DISEÑO DEL SOFTWARE}

Para el desarrollo del sistema de este proyecto se han utilizado software tanto para el desarrollo del programa de control del PLC, para la pantalla táctil (HMI) y para la aplicación del almacenamiento continuo de los datos obtenidos del proceso de destilación de agua.
Así pues para desarrollar el programa del PLC SIEMENS S7-300 se ha empleado el software SIMATIC STEP 7 [2], este software de programación, cuyas principales ventajas son la instalación y configuración sencilla, posee herramientas de programación muy comprensibles, fácil corrección de errores, herramientas para manipular cambios en el PLC mientras entra a RUN y configuración de bloques PID que se usan en este proyecto. En la Fig. 3 se presenta una visión global del software SIMATIC.

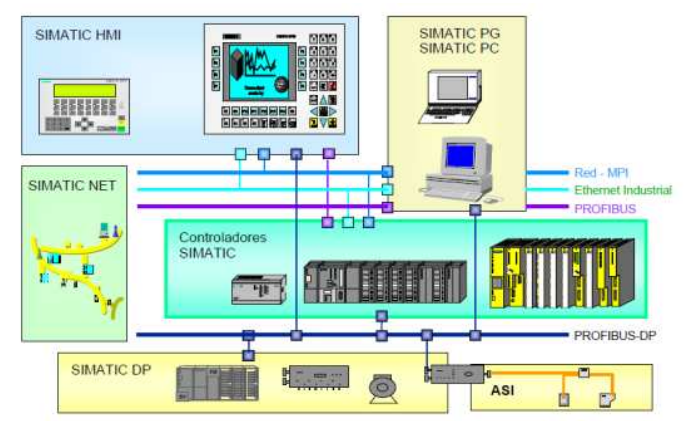

Fig. 3 Visión global del software SIMATIC

Para controlar la variable de presión requerida en el proyecto, el control se realiza con las funciones PID que están integradas en el PLC, en este caso la función que se utilizará es la FB 41 CONT_C (Fig. 4), que sirve para la regulación de procesos industriales con magnitudes de entrada y salida continuas. La función FB 41 CONT_C puede regular fácilmente procesos como el nivel, la presión o el caudal.

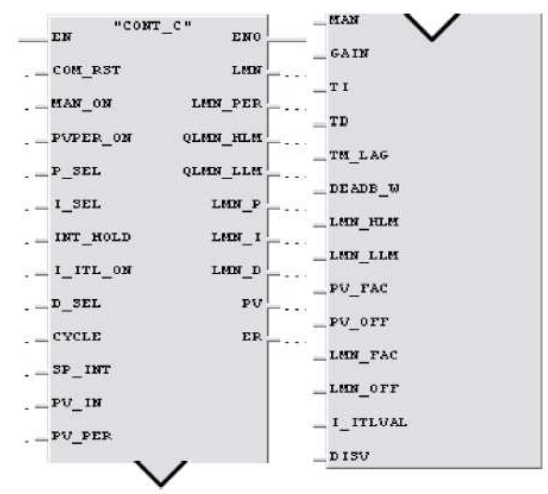

Fig. 4 Aspecto de la función FB 41"CONT_C"

Para la realización de la estación de monitoreo y supervisión se diseño una Interfaz HombreMáquina o HMI ("Human Machine Interface") que es la que permite presentar los datos más relevantes a un operador (humano) (Fig. 5) y a través de la 
cual este controla el proceso, se utilizó el software Wincc Flexible Advanced 2008.

WinCC Flexible [3] también permite la posibilidad de integrar el proyecto del panel de operador al proyecto de Step7 de Siemens, y en nuestro caso, se ha integrado al proyecto de la línea del PLC S7-300 donde hace de interfaz.

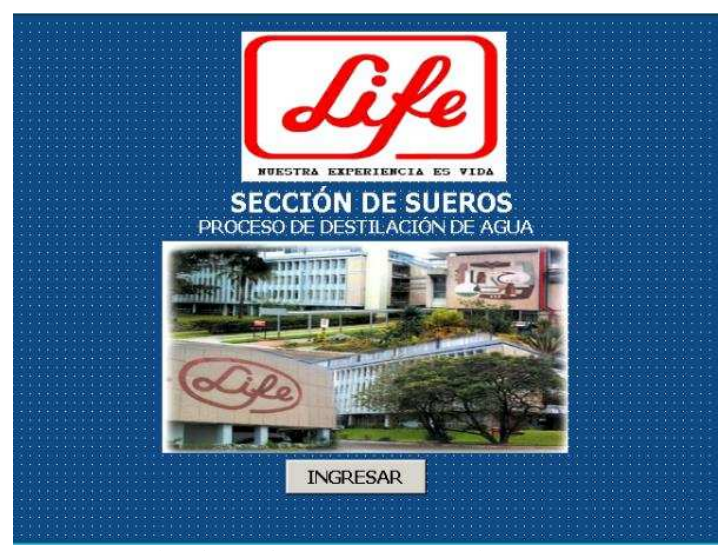

Fig. 5 Interfaz Humano-Máquina (HMI)

El sistema de Monitoreo y Visualización está dividido en once pantallas o ventanas (Fig. 6), en las que están involucradas todas las variables del proceso. En todas las pantallas existen botones que permiten navegar entre ellas.

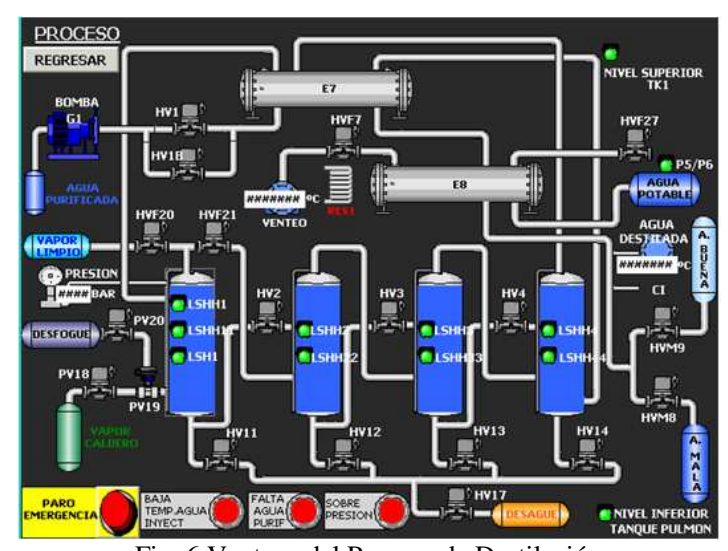

Fig. 6 Ventana del Proceso de Destilación

Para la visualización y control de las variables del proceso (presión y temperatura) en tiempo real se dispone de ventanas con curvas gráficas tal como lo muestran las Figuras 7 y 8.

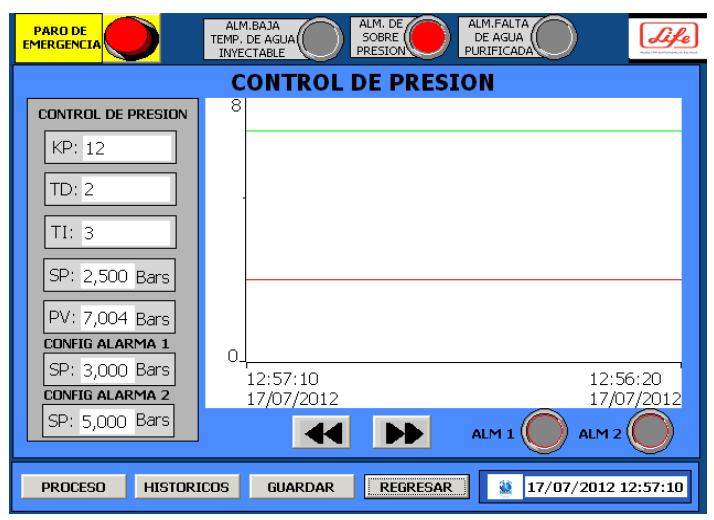

Fig. 7 Ventana de Control de Presión

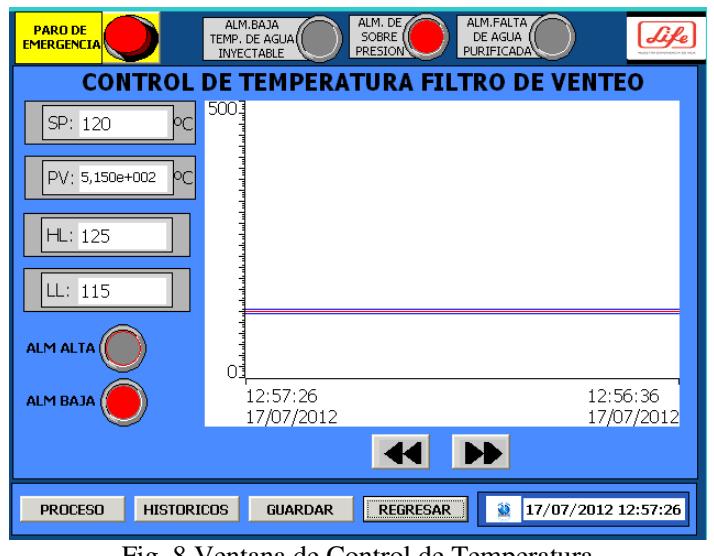

Fig. 8 Ventana de Control de Temperatura

En la ventana configuración de parámetros el operador con la debida autorización puede modificar los valores más relevantes de control para cada una de las variables de proceso (Fig. 9).

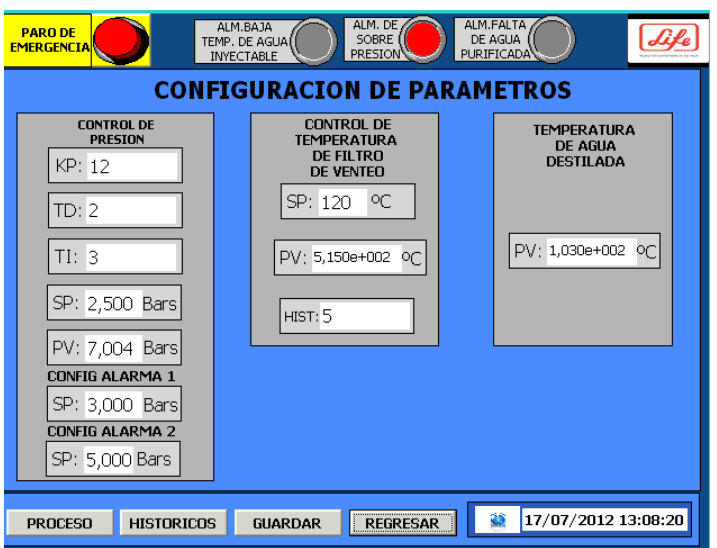

Fig. 9 Ventana de configuración de parámetros

Para la creación de los reportes en hojas de MS Excel (Fig. 10) se utilizó Scripts de WinCC flexible ("Storage_Tag_V1"

"Script_Storage_Path"), los cuales permiten disponer de las variables temperatura y presión. Los datos recopilados del proceso estarán 
disponibles en el computador destinado para el proceso de destilación de agua.

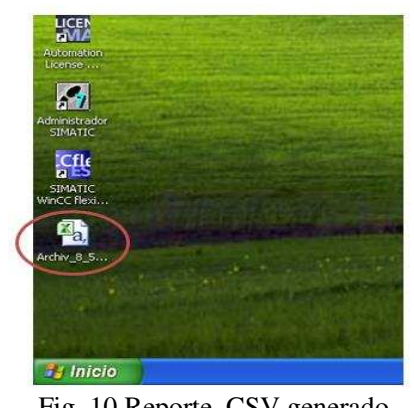

Para la base de datos se ha utilizado el software SQL Server 2005 [4] el cual permitirá almacenar en una tabla todas las variables de proceso más relevantes tal como lo muestra la Fig. 11.

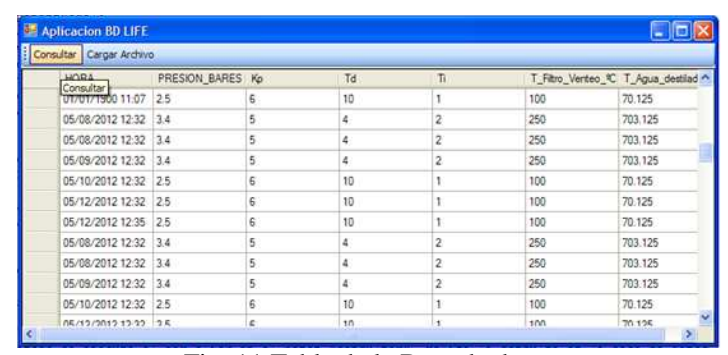

Fig. 11 Tabla de la Base de datos

Para la captura de datos operacionales (Fig. 12) se utiliza el software PC Access el cual permite realizar una comunicación entre el PLC con programas de cómputo tales como: Excel y Visual Basic de esta forma es posible archivar las variables de proceso presión y temperatura en una hora y evaluarlas posteriormente.

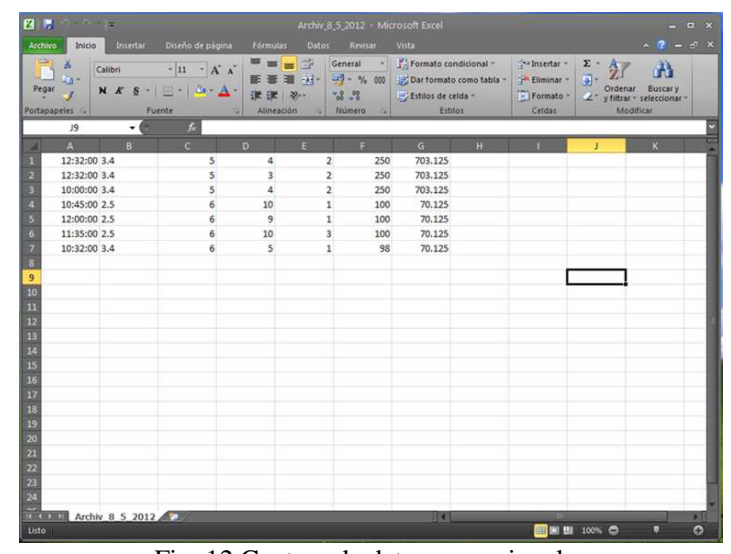

Fig. 12 Captura de datos operacionales

\section{DISEÑO DEL HARDWARE}

El sistema debe ser capaz de controlar todo el proceso de destilación de agua por simple efecto por medio de un PLC el cual gobierna los estados de DESTILACIÓN, GENERACIÓN DE VAPOR, LLENADO, DESCARGA, ABRIR VÁLVULA DE VAPOR Y SANITIZACIÓN, además este controlador debe tomar las señales de los respectivos sensores de nivel, presión y temperatura (Figura 3.1), una vez procesada la información de acuerdo a un modo de control configurado se envían instrucciones a las respectivas electroválvulas que permiten el ingreso de agua y vapor necesarias para los efectos del destilador de agua. Será posible visualizar las variables del proceso y su respectivo control gracias a una pantalla táctil que contendrá una HMI de configuración, sintonización y monitoreo del sistema.

El diseño de cada una de las etapas de hardware con las funciones se describe a continuación:

\section{A. Señales de los sensores}

El destilador OLSA QV 2000 dispone de 9 sensores de nivel, 3 en el primer tanque (Efecto 1), 2 en el segundo tanque (Efecto 2), 2 en el tercer tanque (Efecto 3 ) y 2 en el cuarto tanque (Efecto 4) de estos sensores se registra la señal de 0VDC y 5 VDC (desactivación y activación correspondientemente) para el control de los estados del proceso.

Además dispone de 2 sensores de temperatura, cuyas señales de corriente de $4 \mathrm{~mA}-20 \mathrm{~mA}$ también son registradas, la primera es escalada para la realización del control ON-OFF cuando una válvula de filtro es activada en los estados de Destilación, Llenado, Descarga y Generación de Vapor, la señal del segundo sensor de temperatura en cambio es registrada para indicación en la HMI de la temperatura de agua destilada a la salida del proceso. Por último el destilador cuenta con un sensor de presión cuya señal de 4mA-20 mA es registrada, calibrada y escalada para la realización del control de la válvula de corte de vapor en el estado de Destilación y Generación de vapor.

\section{B. Módulos de entradas y salidas}

De acuerdo a las señales de los respectivos sensores anteriormente mencionados se puede determinar el tipo de módulos a utilizar para registrar dichas señales, así tenemos: Módulos SIEMENS de entradas digitales SM 321, salidas digitales SM 322, entradas analógicas SM 331 y salidas analógicas SM 332. 


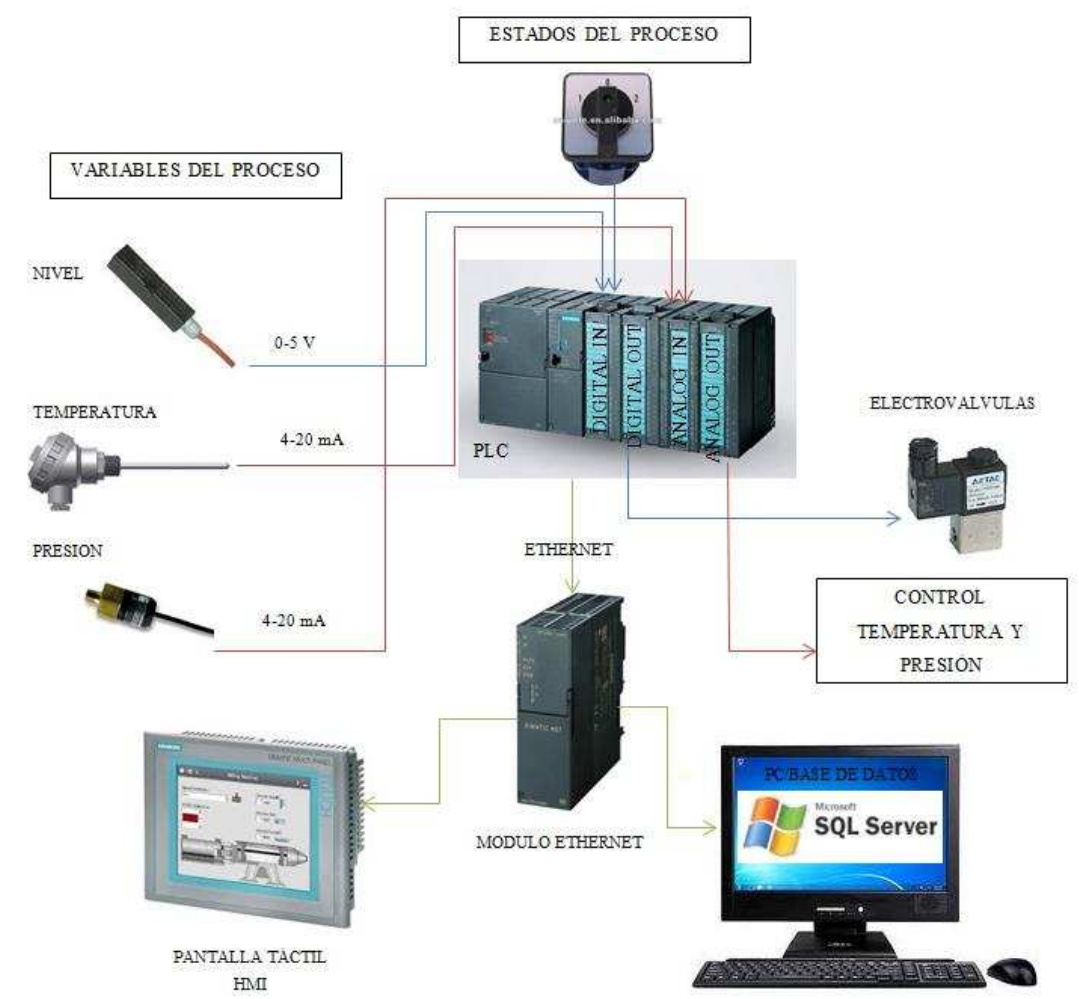

Fig. 13 Etapas de Hardware

\section{PLC SIEMENS S7-300 [5]}

De acuerdo a requerimientos de la empresa y a las condiciones de trabajo donde se va a desarrollar el proyecto el controlador lógico programable empleado será el SIEMENS S7-300, que cumple con todas las necesidades del sistema a desarrollar. Posee un CPU 313C-2DP con un canal de 16 entradas digitales de 24 VDC, alimentación de 24VDC, 16 salidas a relé, y un puerto periférico para conexión de módulos de entradas/salidas y de comunicación.

D. Módulo de comunicación SIEMENS CP 3431 Lean

Dispositivo periférico de la línea SIEMENS, este módulo permite conectar el SIMATIC S7-300 a la red Industrial Ethernet. Como dispone de procesador propio, descarga a la CPU de tareas de comunicación y permite establecer conexiones adicionales la cual permite establecer la transferencia en entornos industriales a largas distancias.

\section{E. Panel Táctil MP 277 10”[6]}

Los Multi Panels (MP) se utilizan como los paneles de operador, para el manejo y la monitorización de máquinas y procesos, además se utilizan en las más diversas ramas y aplicaciones y se pueden ampliar en su aplicación gracias a las opciones Multipanel. La pantalla elegida para este proyecto es una Multipanel de 10 pulgadas con pantalla a color TFT y táctil.

\section{F. Computador}

En el ordenador del proceso se encontrarán funcionando de manera ininterrumpida las aplicaciones para la adquisición y almacenamiento de datos más relevantes (variables del proceso).

Los requerimientos mínimos del ordenador son:

- Procesador: Intel Pentium IV CPU $3.40 \mathrm{GHz}$

- Memoria Ram: 512 MB

- Espacio libre en disco: $20 \mathrm{~GB}$

- Sistema operativo: Windows XP Versión 2002 Service Pack 3

- Software: - Microsoft Office 2007, SQL Server 2005 y Visual Studio 2008

\section{RESUlTADOS}

Para comprobar el correcto funcionamiento del proyecto desarrollado se realizaron dos tipos de pruebas de cada una de las etapas del sistema.

A. Pruebas de hardware

Entre las pruebas realizadas, se han considerado el correcto funcionamiento del 
controlador lógico programable y sus respectivos módulos de entradas/salidas, así como la comunicación entre el autómata, el panel táctil y el ordenador.

En la prueba de funcionamiento del PLC y sus módulos se activaron y calibraron los respectivos sensores y electroválvulas de acuerdo a lo estados del proceso. Estas pruebas se pueden observar en las Figuras 14, 15 y 16.

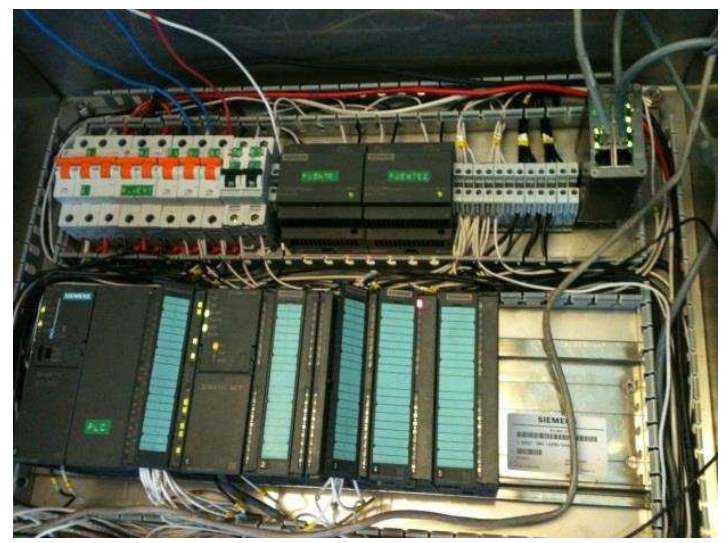

Fig. 14 Pruebas de las señales digitales

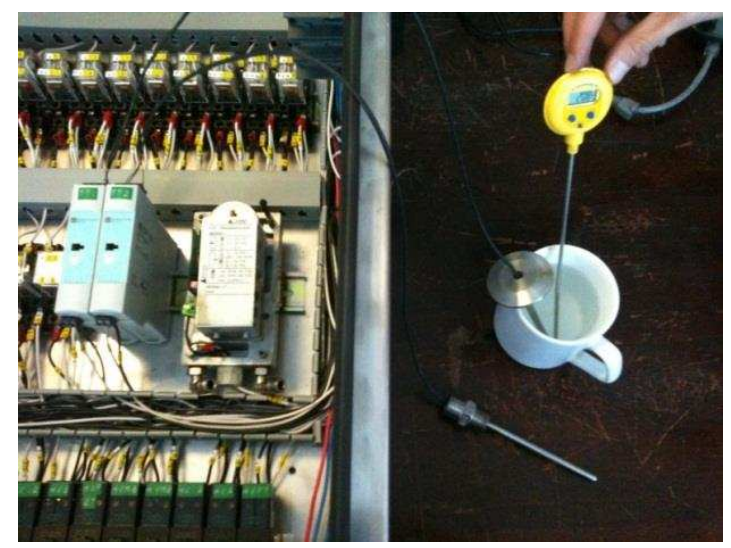

Fig. 15 Calibración de los sensores de temperatura

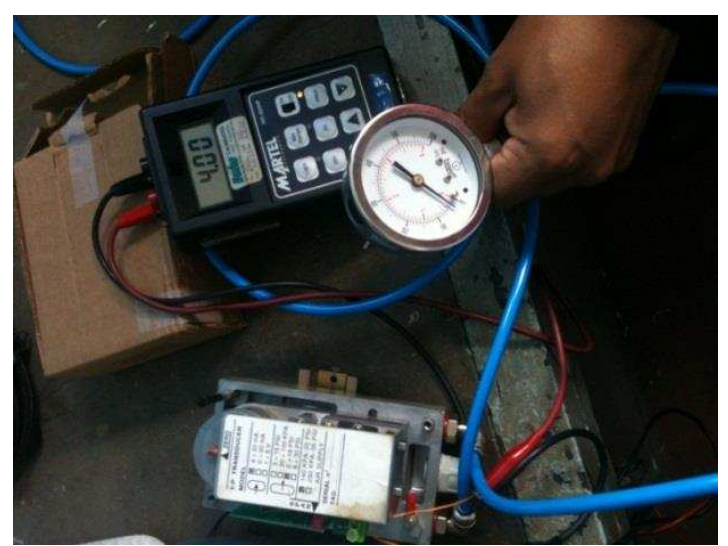

Fig. 16 Calibración del transductor de presión
Para la prueba de comunicación se montó la red Ethernet a través de la conexión del PLC, el panel táctil y el computador a un switch (Fig. 17) asignando a cada uno su respectiva dirección IP. Para verificar que la comunicación se realizó correctamente se ejecuto el comando ping (dirección IP) en el cmd de Windows del PC del proceso para la detección automática de dispositivos.

Este enlace se realizó correctamente y los dispositivos pudieron establecer una comunicación sin interrupciones.

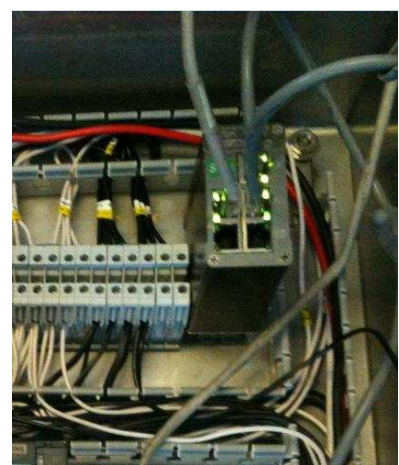

Fig. 17 Comunicación Ethernet entre PLC, PC y Pantalla Táctil

\section{B. Pruebas de software}

En esta prueba se verificó la Interfaz de Monitoreo, Supervisión y Control diseñada para el proceso, así pues se cargo la HMI (Fig. 18) en el panel táctil para comprobación del funcionamiento de las diferentes funciones que van desde los controladores de las variables del proceso (Fig. 19), generación de históricos, hasta la generación de reportes y su almacenamiento en una base de datos (Fig. 20).

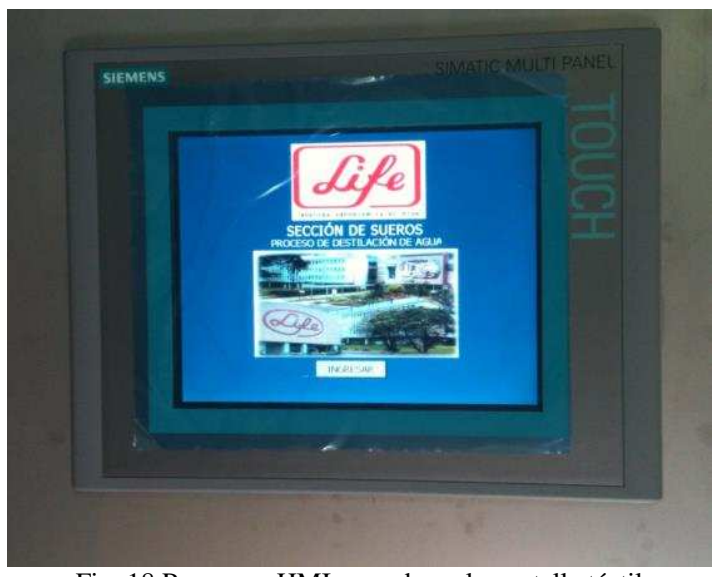

Fig. 18 Programa HMI cargado en la pantalla táctil 


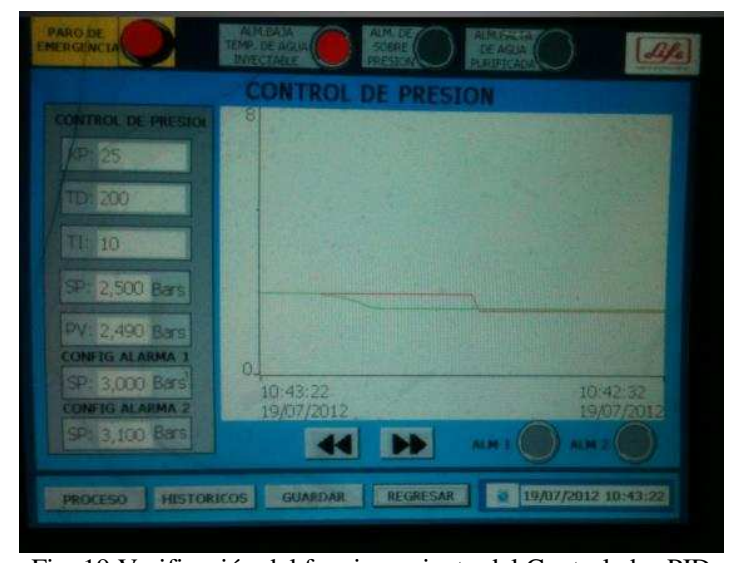

Fig. 19 Verificación del funcionamiento del Controlador PID de Presión

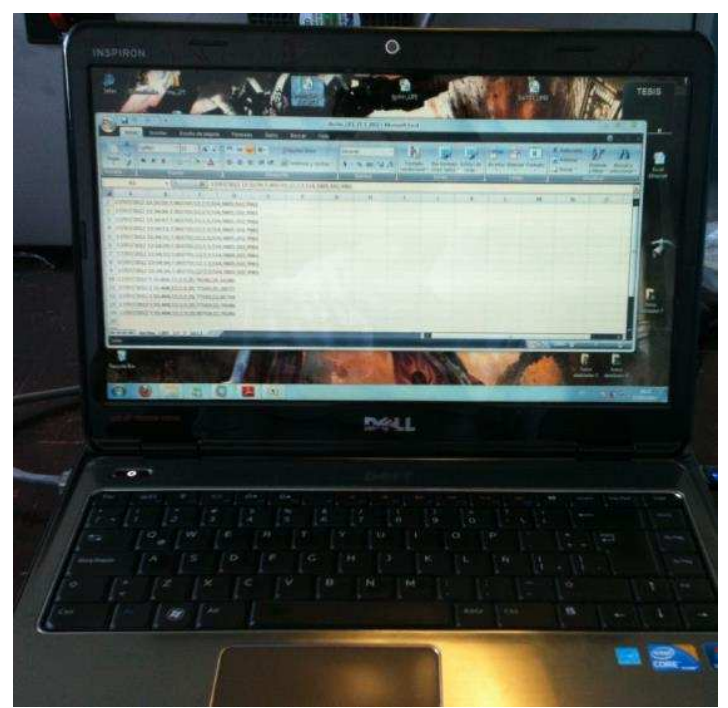

Fig. 20 Pruebas de generación de reportes en MS Excel y base de datos

Todas las pruebas se llevaron exitosa y correctamente permitiendo establecer la validez y aprobación del sistema para su respectiva implementación (Fig. 21).

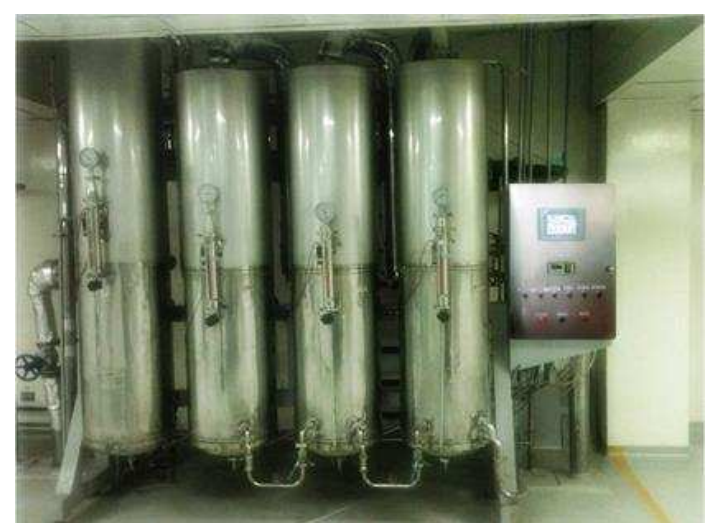

Fig. 21 Nuevo sistema de monitoreo, supervisión y control del proceso de destilación de agua

\section{CONCLUSIONES}

Se desarrolló un sistema de monitoreo, supervisión y control del proceso de destilación de agua empleando un PLC SIEMENS, un panel táctil y software de programación de manera correcta y precisa.

Además, se diseñó una interfaz gráfica sencilla y fácil de entender en una pantalla táctil empleando herramientas de software compatibles que facilita la supervisión, visualización, monitoreo en tiempo real y control de operación de los equipos del proceso de destilación de agua.

Se configuraron y sintonizaron de manera precisa el controlador PID de presión y el controlador de temperatura del filtro de venteo según las necesidades del proceso.

A través de la creación de reportes en hojas de cálculo en Excel y la base de datos se facilitó el análisis de los valores registrados de las variables del proceso, además resultaron herramientas muy importantes para los operadores ya que permiten incrementar y mejorar los niveles de producción.

\section{VII.REFERENCIAS}

[1] Pagina Oficial Laboratorios Farmacéuticos del Ecuador LIFE C.A. [Online]. Habilitado:

http://laboratorioslife.com/templates/life/_html/productos.html

[2] Software para controladores SIMATIC [Online].

Habilitado:

http://www.automation.siemens.com/salesmaterialas/brochure/es/brochure simatic-industrial-software_es.pdf

[3] SIMATIC HMI WinCC flexible 2008 Compact / Standard / Advanced.pdf

[4] Cómo hacer una base de datos sencilla en SQL SERVER 2005... [Online]. Habilitado: http://jotask8punk.wordpress.com/otras-tecnologias/comohacer-una-base-de-datos-sencilla-en-sql-server$2005 \%$ E2\%80\% A6/

[5] Sistemas de automatizacion industrial SIMATIC [Online]. Habilitado:

http://www.distribuidor-oficial-siemens-productoselectricos.control-technics.com.ar/distribuidorsiemens/es/Notas\%20y\%20articulos/Automatizacion/SIMATI C/1682/Sistemas\%20de\%20automatizacion\%20industrial\%20 SIMATIC.htm

[6] SIMATIC HMI Panel de operador MP 277 (WinCC flexible) Instrucciones de servicio.pdf 